G. F. Bottazzo • E. Bosi - C. A. Cull - E. Bonifacio •

M. Locatelli · P. Zimmet • I. R. Mackay · R. R. Holman

\title{
IA-2 antibody prevalence and risk assessment of early insulin requirement in subjects presenting with type 2 diabetes (UKPDS 71)
}

Published online: 19 May 2005

(C) Springer-Verlag 2005

The online version of the original article can be found at http://dx. doi.org/10.1007/s00125-005-1691-9

G. F. Bottazzo · M. Locatelli

Scientific Directorate, Bambino Gesù Paediatric Hospital,

Scientific Institute,

Rome, Italy

E. Bosi - E. Bonifacio

General Medicine, Diabetes and Endocrinology Unit,

San Raffaele Vita-Salute University Hospital,

Milan, Italy

C. A. Cull $(\bowtie) \cdot$ R. R. Holman

Diabetes Trials Unit, Oxford Centre for Diabetes,

Endocrinology and Metabolism, Churchill Hospital,

Old Road, Headington,

Oxford, OX3 7LJ, UK

e-mail: carole.cull@dtu.ox.ac.uk

Tel.: +44-1865-857251

Fax: +44-1865-857254

P. Zimmet

International Diabetes Institute,

Caulfield, Victoria, Australia

I. R. Mackay

Department of Biochemistry and Molecular Biology, Monash University,

Clayton, Victoria, Australia

\section{Diabetologia (2005) 48:703-708}

Unfortunately there was a mistake in the sub-section of the Results entitled 'IA-2A, ICA, GADA and early requirement for insulin therapy'. The last sentence reads:

Patients older than 45 years at diagnosis were more likely to require insulin than those aged 45 years or less (23.4 vs $7.8 \%$ respectively, $p<0.00001$ ).

It should read:

Patients younger than 45 years at diagnosis were more likely to require insulin than those aged 45 years or more (23.4 vs $7.8 \%$ respectively, $p<0.00001$ ). 